\title{
Risk Management of Assets Dependency Based on Copulas
}

\section{Function}

\author{
Lei Cheng and Xiaofang Chen \\ School of Management, Wuhan University of Technology, Wuhan, China \\ E-mail: chenglei_cl@126.com,cxf2010611@163.com
}

\begin{abstract}
As the two important form of financial market, the risk of financial securities, such as stocks and bonds, has been a hot topic in the financial field; at the same time, under the influence of many factors of financial assets, the correlation between portfolio returns causes more research. This paper presents Copula-SV-t model that it uses SV-t model to measure the edge distribution, and uses the Copula-t method to obtain the high-dimensional joint distribution. It not only solves the actual deviation with using the ARCH family model to calculate the portfolio risk, but also solves the problem to overestimate the risk with using extreme value theory to study financial risk. Through the empirical research, the conclusion shows that the model describes better assets and tail characteristics of assets, and is more in line with the reality of the market. Furthermore, Empirical evidence also shows that if the portfolio is relatively large degree of correlation, the ability to disperse portfolio risk is relatively weakness.
\end{abstract}

Keywords. Portfolio, Correlation analysis, Copulas function, Risk management.

\section{Introduction}

Economic globalization and financial integration greatly enhanced the interdependence between the global economy and financial markets; The synergy of prices in financial markets made local fluctuations of any areas have an influence on other financial markets, triggered the financial crisis. During the Asian financial crisis in 1997, Hengsheng index in Hong Kong dropped from the top of the nearly 16000 point to less than 8000 points. And during the global financial crisis in 2007, the subprime crisis originated in America spread to the global market. A-share index fell from 6200 to 2000, at the same time, Hengsheng index also fell from 30000 to 12000, indicating a strong following up. So the study of the dependent features and risk factors among the assets structure is an on-going process in the financial risk analysis.

For foreign references [1, 2, 4], Frees, Valdez and Embrechts studied simulation and estimation of individual asset dependency structure between among time series, without studying multiple assets dependency structure. Nelsen introduced the Copulas function into the finance, and discussed the limitation of using the linear correlation index to measure the dependency in the financial markets. Furthermore he suggested to estimate the joint distribution with Copulas theory among the random variables. Domestic study of Copulas connection theory started late $[9,10,11]$. Zhang Yaoting (2002) theoretically discussed the feasibility of the application of Copulas function in the financial industry, and pointed out that using Copulas technology to analyze the correlation structure between the variables can be more reliable between 
the variables. Wei YanHua and Zhang Shiying (2004) applied the Copulas to GARCH model to measure the autocorrelation structure of financial time series.

The above research either concerns with a single portfolio risk, or the static study of portfolio. In order to study the relationship of portfolios, these research has been neither conducted from the perspective of dynamic effect, nor considered assets as additive and independent. This paper will introduce Copulas function to study dependency structure of multiple assets, to measure the correlation between each other, and to verify the correlation with the empirical analysis. Therefore, not only the effective analysis of assets dependency will be achieved, but also the purpose of forecast and control of the financial risk will be attained.

\section{The tail correlation and copulas function}

Financial market is full of risk, but Markowitz creatively put forward that diversified investment can reduce risk level under a certain range. In general, investors will choose assets of small correlation to reduce investment risk. So correlation analysis of assets portfolio has great influence on the returns, which needs further interpretation.

Copulas function does not contain any information about marginal distribution, but contains relevant information between marginal distribution. This correlation is often non-linear. Generally we use Kendalltor Spearmanprank correlation coefficient to measure the correlation of marginal distribution. Because correlation coefficient has a strong robustness, and is strongly resistant to the impact of abnormal data. Therefore it has been used to measure the correlation. $\mathrm{T}$ is defined as the difference of two random variables between the same direction and the reverse direction.

Definition: if $\left(x_{1}, y_{1}\right),\left(x_{2}, y_{2}\right)$ is the vector of independent and identically distributed, $x_{1}, x_{2} \in x, y_{1}, y_{2} \in y$,

$$
\tau=p\left\{\left(x_{2}-x_{1}\right)\left(y_{2}-y_{1}\right)>0\right\}-p\left\{\left(x_{2}-x_{1}\right)\left(y_{2}-y_{1}\right)<0\right\}
$$

then $\tau$ measures the consistency of the variability of $\mathrm{x}$ and $\mathrm{y}$, which proves

$$
\tau=2 p\left\{\left(x_{2}-x_{1}\right)\left(y_{2}-y_{1}\right)>0\right\}-1, \quad \tau \in[-1,1]
$$

According to research and deduction of Kendall's rank correlation coefficient, we can get the following conclusion: $\tau$ changes reflect the extent of consistency of the variability of $x$ and $y$.

Table 1 shows two Copulas functions with only one parameter, and if the estimated value of $\tau$ is calculated, the whole nature of Copulas function is sure to know.

Table 1. The Copula function parameters and the correlation coefficient.

\begin{tabular}{|c|c|c|c|}
\hline Type & Copula distribution & $\theta$-Range & $\tau$-Parameter \\
\hline Gumbel & $\exp \left\{-\left[(-\ln u)^{1 / \theta}+(-\ln v)^{1 / \theta}\right]\right.$ & {$[1, \infty)$} & $\theta /(\theta+2)$ \\
\hline Clayton & $\left(u^{-\theta_{+}} v^{-\theta}-1\right)^{-1 / \theta}$ & {$[-1,0) \bigcup(0, \infty)$} & $\theta /(\theta-2)$ \\
\hline
\end{tabular}

\section{Single asset yield model (garch $-t)$}

When we use Copulas technology to build the model, we can separate correlation structure between marginal distribution and random variable. This will help us to analysis and understand a lot of financial problems; At the same time, for conditional heteroscedasticity and tailing characteristics of the financial time series, the following research will analyze financial time series model of single variable distribution---- building of marginal function.

Traditional fluctuation model believes that exogenous variables, such as the exchange rate or interest rate, can cause yield heteroscedasticity (volatility). But conditions mean and variance may change by time, leading to the change of the 
variance. So Engel (1982) [2] proposed conditional heteroscedasticity (ARCH) model to describe the time series with the conditions of the second order moment in nature, and describe fluctuations of time-varying and aggregation through the change of conditional heteroscedasticity. In the ARCH model, Engel points out that the mean $\varepsilon_{t}$ interference conditions do not change by time. But in the ARCH model, variance $\left(h_{t}\right)$ depends on the time series of past observations. So according to the description of the ARCH model, under the condition of known information, $y_{t}$ obey normal distribution where $\varepsilon_{t}=0, h_{t}$. Thus the ARCH model can be used to describe the condition of variable marginal distribution.

Because in practical application the ARCH model takes a lot of order, leading to deficiency in calculation, Bollerslev (1986) proposed the Generalized regressive conditional heteroscedasticity (GARCH) model, which is an important extension of the ARCH model. The model has faster lagged convergence than the ARCH model, and the similar structure with the ARMA model.

$\operatorname{GARCH}(\mathrm{p}, \mathrm{q})$ - T model is defined as follows

$$
\left\{\begin{array}{l}
r_{t} \mid I_{t-1} \sim \mathrm{t}_{v} \\
h_{t}=\partial_{0}+\sum_{i=1}^{q} \partial_{i} \varepsilon_{t-i}^{2}+\sum_{i=1}^{p} \beta_{i} h_{t-i} \\
r_{t}=h_{t}^{1 / 2} v_{t}
\end{array}\right.
$$

Where, $r_{t}$ is the series of return, $p \geq 0, q>0 ; \partial_{0}>0$,

$\partial_{i} \geq 0, i=1, \ldots, q ; \beta_{i} \geq 0, i=1, \ldots, p_{\circ} \mathrm{IF} \mathrm{p}=0$, GARCH process becomes ARCH (q) process. So, you can consider ARCH process as a special case of GARCH process.

\section{Multi-asset portfolio model and parameter estimation}

\subsection{Construction of multi-asset portfolio model}

Supposing, portfolio have $\mathrm{n}$ kind of financial assets, $i(i=1,2, \ldots, n)$, and build model directly by using GARCH - $\mathrm{t}$ model, according to the latest $\mathrm{m}$ phase of the historical data

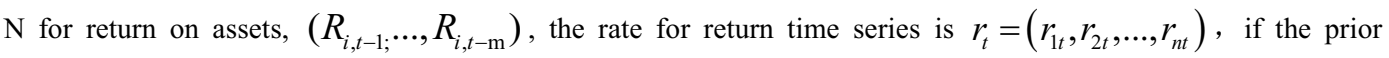
information set before $t$ moment shows

$$
I_{t-1}=\left\{r_{t-1}, h_{t-1}, r_{t-2}, h_{t-2}, \ldots\right\}=\bigcup_{i=1}^{n} I_{i, t-1} \quad, \quad I_{i, t-1}=\left\{r_{i, t-1}, h_{i, t-1}, r_{i, t-2}, h_{i, t-2}, \ldots\right\} \quad, \quad h_{i t} \text { is conditional }
$$

volatility for the information set $\left(I_{i, t-1}\right)$ of $\operatorname{singular} \operatorname{asset}\left(r_{i t}\right), C\left(\cdot \mid I_{t-1}\right)$ shows Copulas function for $\mathrm{n}$, $F_{i}\left(r_{i, t} \mid I_{i, t-1}\right)$ is the conditional distribution of i-th component

Many empirical studies have shown that asset return sequence obeyed the GARCH $(1,1)$ model. Bollerslev (1987) point out that $\mathrm{t}$-student distribution can reflect more characteristics for financial time series than the normal distribution 
can do, such as the peak of time series and thick tail. Here we also assume that $r_{i t}$ satisfy the model. After filteried the fluctuation of time-varying about a single series, we propose a time-varying model ---Copulas- GARCH $(1,1)$ - $t$ (shorthand for Copulas- GARCH - $\mathrm{t}$ ). The term used to describe the time-varying dependence structure of $\mathrm{n}$ assets, and the model is as follow:

$$
\left\{\begin{array}{l}
r_{i, t}=u+\varepsilon_{i, t} \\
\varepsilon_{i, t}=\sqrt{h_{i, t}} \cdot v_{i, t} \\
h_{i, t}=\alpha_{0}+\alpha_{1} \varepsilon_{i, t-1}^{2}+\beta_{1} h_{i, t-1} \\
F\left(v_{t} \mid I_{t-1}\right)=C_{\Sigma}\left[F_{1}\left(v_{1, t} \mid I_{1, t-1}\right), F_{2}\left(v_{2, t} \mid I_{2, t-1}\right), \ldots, F_{n}\left(v_{n, t} \mid I_{n, t-1}\right) \mid I_{t-1}\right]
\end{array}\right.
$$

where, $r_{i t}$ is the series for return, $u$ is the average return rate for singular asset, $\quad p \geq 0, q>0 ; \partial_{i} \geq 0, \beta_{i} \geq 0$;

$F\left(v_{t} \mid I_{t-1}\right)$ is the joint distribution portfolio on the moment of T, $C_{\Sigma}$ is Copula function. $\alpha_{0}, \alpha_{1}, \beta, v$ and $\sum$ are parameters to be estimated.

\section{2 Parameter estimation of Copulas - GARCH function}

Parameter estimation of function model includes parameter estimation and non-parameter estimation. Parameter estimation method generally adopts maximum likelihood estimation (MLE) and the torque estimation. The maximum likelihood estimation is the most commonly and most effective method of Copulas function model. Parameters $\alpha_{0}, \alpha_{1}, \beta_{1}$ to be estimated in the model for single asset are obtained through the Eview software, and the parameters of the portfolio will be obtained by maximum likelihood estimation.

If we set random variables $x_{1}, \ldots, x_{N}$ in the Copula function as $C(., \ldots,$.$) , density distribution is \mathrm{c}(., \ldots,$.$) , the$ marginal distribution $F_{n}(.,$.$) , the edge density function f_{n}(.,),. \mathrm{n}=1, \ldots, \mathrm{N}$, then the parameters $\theta=\left(\theta_{1}, \ldots, \theta_{N} ; \theta_{c}\right)$ of the logarithm likelihood function is:

$$
\left.\ln L\left(x_{1}, \ldots x_{N} ; \theta\right)=\sum_{t=1}^{T}\left[\ln c\left(F_{1}\left(x_{1} ; \theta_{1}\right), \ldots, F_{N}\left(x_{N} ; \theta_{N}\right), \theta_{C}\right)\right)+\sum_{n=1}^{N} \ln f_{n}\left(x_{n, t}, \theta_{n}\right)\right]
$$

We can directly use the maximum likelihood estimation for parameter estimation. But from the logarithmic likelihood function we can find that the marginal distribution density and the density of Copulas likelihood function is separate. Therefore, we can first estimate the edge parameter, then the Copulas function parameter. The estimation of parameter is divided into two phases.

The first phase: $\quad \hat{\theta}_{n}=\arg \max \sum_{t=1}^{T} \ln f_{n}\left(x_{n, t}, \theta_{n}\right), \mathrm{n}=1, \ldots, \mathrm{N}$

The second phase: $\hat{\Sigma}_{c}=\arg \max \sum_{t=1}^{T} \ln c\left(F_{1}\left(x_{1, t} ; \hat{\theta}_{1}\right), \ldots, F_{N}\left(x_{N} ; \hat{\theta}_{N}\right), \theta_{C}\right)$ 
First estimate the parameter in the marginal distribution $\theta_{n}, n=1, \ldots, N$, and then put the estimated value $\hat{\theta}_{n}$ into Copula function of different kinds, thus estimate the parameter of Copula function $\hat{\Sigma}_{c}$.

\subsection{The empirical analysis}

Here we only analyze two stock market index of daily closing price: Baoli Real Estate and Wanke Real Estate; the data range from July 31, 2006 to May 9, 2013. It is important to note that because there may be one day when two stocks are not trading at the same time, in order to accurately describe the correlation of the two stocks, we consider data observed as the data for that day when only one stock has dealings ( $\mathrm{t}$ stands for trading day). After pretreatment, valid data of observation samples reach 1372, namely $\mathrm{T}=1372$. Secondly, the daily closing price is selected as poor stock index, logarithmic LPR, defined as LPR $=\log (\mathrm{pr})-\log (\mathrm{pr}(1))$.

(1) The figure of the return rate of the two stocks, in which bp stands for Baoli Real Estate's daily yield rate, wp stands for Wanke Real Estate closing price.
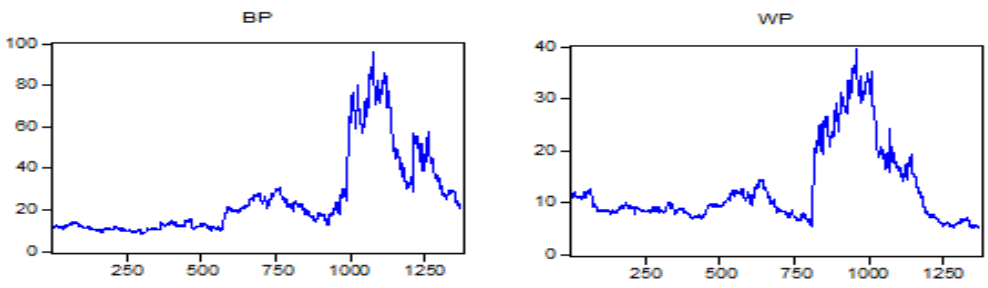

Fig. 1. The daily yield rate of the two stocks.

(2) Descriptive statistics of the two stocks

Table 2. Descriptive statistics of the two stocks.

\begin{tabular}{|l|l|l|l|l|l|l|l|l|l|}
\hline & Mean & Median & Maxi & Min & Std. Dev & Skewness & Kurtosis & J-Bera & Prob \\
\hline & 25.715 & 19.055 & 96.23 & 8.52 & 19.52 & 1.605 & 4.654 & 745.244 & 0 \\
\hline & & & & & & & & & \\
\hline & 12.632 & 9.3 & 39.5 & 4.93 & 7.60 & 1.578 & 4.511 & 699.837 & 0 \\
\hline
\end{tabular}

From the table, we can draw the following analysis. During the sample period, the mean of bp stands at 25.7, standard deviation 19.5 with 1.6 of skewness, and 4.7 kurtosis. Compared with the normal distribution, bp has the tendency to slide right with rush thick tail phenomenon. $\mathrm{JB}$ normality test also confirmed this, with $\mathrm{JB}=745.2, \mathrm{P}=0$, and refused null hypothesis. These show that under the confidence level $\partial=0.05$, Baoli Real Estate significantly differs from those of normal distribution. Similarly, Wanke Real Estate also has the tendency to slide right with rush peak phenomenon and significantly differs from those of normal distribution.

(3) Parameter estimation

Table 3. Two property parameter estimation.

\begin{tabular}{|c|c|c|c|c|c|c|}
\hline & $u$ & $\partial_{0}$ & $\alpha_{1}$ & $\beta_{1}$ & $v$ & $\sum_{c}$ \\
\hline lbp & $\begin{array}{l}1.000002 \\
(2525.385)\end{array}$ & $\begin{array}{l}0.0000134 \\
(3.499094)\end{array}$ & $\begin{array}{l}0.004938 \\
(2.740976)\end{array}$ & $\begin{array}{l}0.983879 \\
(239.6179)\end{array}$ & $\begin{array}{l}3.872199 \\
(12.86543)\end{array}$ & \multirow{2}{*}[\begin{array}{l}{1,0.078442}\\
{0.078442,1}\end{array}]{} \\
\hline lwp & 0.999609 & 0.001052 & 0.103511 & -0.00604 & 3.433089 & \\
\hline
\end{tabular}




\begin{tabular}{|l|l|l|l|l|l|l|}
\hline & $(1946.871)$ & $(9.165047)$ & $(2.179955)$ & $(-0.18711)$ & $(12.11436)$ & \\
\hline
\end{tabular}

From the above table, we know that $\alpha_{1}$ and $\beta_{1}$ in logarithm return rate (LBP) is respectively equal to 0.004938 and 0.983879 in the conditional variance equation, and they are significantly different. It shows that the return series has significant volatility cluster. Secondly, in Baoli Real Estate's LBP, the sum of ARCH and GARCH coefficients equals to 1 , so the GARCH $(1,1)$ process is stable, that is, the effects of fluctuations in the past on the future is gradually fading. Similarly, we can get logarithm yield values of the parameters for Wanke Real Estate.

(4) Equation residual test

\begin{tabular}{|c|c|c|c|c|c|c|}
\hline Autocorrelation & Partial Correlation & & $A C$ & PAC & Q-Stat & Prob \\
\hline 小 & 小 & & -0.000 & -0.000 & 0.0003 & 0.987 \\
\hline | 1 & | & & 0.004 & 0.004 & 0.0221 & 0.989 \\
\hline 小 & 1 1 & & -0.002 & -0.002 & 0.0266 & 0.999 \\
\hline d & d & & -0.030 & -0.030 & 1.2946 & 0.862 \\
\hline 11 & 1 & & -0.024 & -0.024 & 2.0710 & 0.839 \\
\hline 小 & 小' & & -0.002 & -0.002 & 2.0791 & 0.912 \\
\hline d & d & & -0.030 & -0.030 & 3.3004 & 0.856 \\
\hline 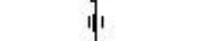 & 11 & 8 & -0.009 & -0.010 & 3.4130 & 0.906 \\
\hline$\|_{1}$ & | & 9 & 0.001 & -0.000 & 3.4140 & 0.946 \\
\hline 川 & 1 & 10 & -0.020 & -0.021 & 3.9806 & 0.948 \\
\hline
\end{tabular}

Fig. 2. Baoli Real Estate autocorrelation.

The residual test of equation shows $\mathrm{Q}$ statistic of the logarithm return rate on Baoli Real Estate, at $\mathrm{k}=10, \mathrm{p}=0.948$. It rejects the original hypothesis, namely the residual sequence is independent from each other, which meets the requirements.

\begin{tabular}{|c|c|c|c|c|c|c|}
\hline Autocorrelation & Partial Correlation & & $A C$ & PAC & Q-Stat & Prob \\
\hline 小 & 小 & & -0.001 & -0.001 & 0.0006 & 0.980 \\
\hline d & d & & -0.031 & -0.031 & 1.3540 & 0.508 \\
\hline 少 & in & & 0.011 & 0.011 & 1.5218 & 0.677 \\
\hline in & $\sqrt{1}$ & & 0.008 & 0.007 & 1.6148 & 0.806 \\
\hline i & ii & & -0.010 & -0.010 & 1.7591 & 0.881 \\
\hline 小 & 小 & 6 & 0.006 & 0.006 & 1.8056 & 0.937 \\
\hline 11 & $\pi$ & & -0.019 & -0.020 & 2.2974 & 0.942 \\
\hline 中 & 中 & 8 & 0.016 & 0.017 & 2.6506 & 0.954 \\
\hline 小 & 小 & 9 & 0.001 & -0.000 & 2.6516 & 0.977 \\
\hline 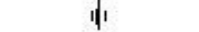 & | & 10 & -0.009 & -0.007 & 2.7562 & 0.987 \\
\hline
\end{tabular}

Fig. 3. Wanke Real Estate autocorrelation.

The residual test of equation shows $\mathrm{Q}$ statistic of the logarithm return rate on Wanke Real Estate at $\mathrm{k}=10, \mathrm{p}=0.987$. It rejects the original hypothesis, namely the residual sequence is independent from each other, which meets the requirements.

\section{Conclusion}

Based on the conditional heteroscedasticity GARCH-t model for portfolio, this thesis attempts to simulate spike and thick tail characteristics, and describes the characteristics of time-varying and state independence of the distribution on financial assets, and solves the problem proposed in the beginning; By using Copulas function, the asset correlation is reflected, and the portfolio selection problem is solved in the risk management. And from an empirical analysis of the Baoli Real Estate and Wanke Real Estate, the following conclusion can be drawn:

Conclusion 1: There are significant GARCH effects in two stocks, namely the conditional heteroscedasticity. Positive risk premium of Baoli is higher than that of Wanke, which reflects the Baoli stock investors are more risk-averse than Wanke investors. 
Conclusion 2: There is obvious leverage in two stocks, which the effect of bad news on the stock market will cause more volatility than that of the good news. For example, in 2008, after increasing the stamp duty, there was a sharp decline in stock market. There was two-way spillover effect between the two stocks, which indicates that a stock fluctuations can cause the fluctuation of the other stock.

\section{References}

1. Engle,R.E. Dynamic Conditional Correlation:A Simple Class of Multivariate Generalized Autoregressive Conditional Heteroskedasticity Models. Journal of Business\&Economic Statistics, 2002,7:339-350.

2. J.C.Rodriguez. Measuring Financial Contagion: A Copula Approach, Journal of Empirical Finance, 2003,14: 401-423.

3. Embrechts P, Hoeing A, Juri A. Using copula to bound the value at Risk for functions of dependent risks . Finance and Stochastics, 2003, 7:145- 167.

4. Fan J·, Jiang, J·Zhang, etal, Time-Dependent Diffusion Models for Term Structure Dynamics, Statistica Sinica, 2003, 13: 965 992.

5. Chen M, Chen G. A nonparametric test of conditional autoregressive heteroscedasticity for threshold autoregressive models. The Canadian Journal of Statistics, 2008,4:649 - 666.

6. R B Nelsen·,An Introduction to Copulas, Springer, New York,2006:51-155.

7. ZHANG Xuegong,XUE Zhichao,etal. Analysis of financial market based on Copula-SV-t Pair-Correlation, journal of Finance and Economics, 2016.04:71-76 (In Chinese).

8. YI Wen-de.Conditional dependence modelsof assets based on and its applications of portfolios Copula functions.Systems Engineering-Theory and Practice, 2011, 6:1004-1013 (In Chinese).

9. WEN X ,WEI Y,HUANG D.Measuring contagion between energy market and stock market during financial crisis:a copula approach. Energy economics,2012,34(5):1435-1446(In Chinese).

10. Kunlapath S,Tatevik Z,etal.Interdependence of oil pricess and stock market indices:a copula approach.Energy Economics, 2014,44(4):331-339.

11. YAO Den-bao,LIU Xiao-xing,7HANG Xu.Dynamic Correlation Structure Between Market Liquidity and Market Expectation:An Analysis Based on ARMA-GJR-LARCH-Copula Model, Chinese Journal of Management Science, 2016.2 (4):1-10(In Chinese).

12. YU Wen-hua.Study on Extreme Value Dependence Measurement of Shanghai and Shenzhen Composite Index-Based on the Four Types of Time-Varying Copula Model,Journal of southwest jiaotong university2013,7(4):90-96(In Chinese). 